\title{
Polarization switching phenomena in semipolar InxGa1-xN/GaN quantum well active layers
}

\section{AUTHOR(S):}

Ueda, M; Funato, M; Kojima, K; Kawakami, Y; Narukawa, Y; Mukai, T

\section{CITATION:}

Ueda, M ...[et al]. Polarization switching phenomena in semipolar InxGa1-xN/GaN quantum well active layers. PHYSICAL REVIEW B 2008, 78(23): 233303.

\section{ISSUE DATE:}

2008-02

URL:

http://hdl.handle.net/2433/84618

RIGHT:

(C) 2008 The American Physical Society 


\title{
Polarization switching phenomena in semipolar $\operatorname{In}_{x} \mathrm{Ga}_{1-x} \mathrm{~N} / \mathrm{GaN}$ quantum well active layers
}

\author{
M. Ueda, ${ }^{1}$ M. Funato, ${ }^{1, *}$ K. Kojima, ${ }^{1}$ Y. Kawakami, ${ }^{1}$ Y. Narukawa, ${ }^{2}$ and T. Mukai ${ }^{2}$ \\ ${ }^{1}$ Department of Electronic Science and Engineering, Kyoto University, Kyoto 615-8510, Japan \\ ${ }^{2}$ Nitride Semiconductor Research Laboratory, Nichia Corporation, Tokushima 774-8601, Japan \\ (Received 14 August 2008; revised manuscript received 2 October 2008; published 8 December 2008)
}

\begin{abstract}
We report the observation of optical polarization switching in $\operatorname{In}_{x} \mathrm{Ga}_{1-x} \mathrm{~N} / \mathrm{GaN}$ quantum well active layers, using semipolar $\{11 \overline{2} 2\}$ planes. When the In composition is less than $\sim 30 \%$, the emissions related to the top and second valence bands are polarized along the [1 $\overline{1} 00]$ and perpendicular [ $\overline{1} \overline{1} 23]$ directions, respectively, similar to earlier studies. On the contrary, as the In composition increases above $30 \%$, the polarizations switch, indicating a crossover between the two valence bands. Because the polarization degree is less sensitive to the well width, the observed polarization switch is ascribed to the $\mathrm{InN}$ deformation potentials.
\end{abstract}

DOI: 10.1103/PhysRevB.78.233303

A typical nitride-semiconductor-based light emitter consists of $c$-oriented quantum wells (QWs), where piezoelectric and spontaneous polarizations lower the optical transition probability. ${ }^{1}$ One way to circumvent this decreased probability is to grow QWs on nonpolar ${ }^{2,3}$ or semipolar ${ }^{4-7}$ planes tilted from the $c$ plane because a reduced or even negligible polarization is expected. ${ }^{8,9}$ Recently, we have experimentally demonstrated that the $\{11 \overline{2} 2\}$ plane is promising for high emission efficiencies ${ }^{4,5}$ and have fabricated light-emitting diodes (LEDs). ${ }^{6}$

Unlike the polar (0001) plane, nonpolar and semipolar planes have low crystal symmetries, which cause optical anisotropy. For example, because the lowest energy transition in $\mathrm{GaN}$ is polarized perpendicular to the [0001] direction, polarization is not observed from the [0001] direction, whereas linear polarization is observed from the nonpolar directions. Intermediately, the polarization degree in unstrained, semipolar $\{11 \overline{2} 2\} \mathrm{GaN}$ has been calculated to be 56\% (Refs. 10 and 11) and has been experimentally confirmed to be $46 \%$ by photoluminescence (PL) and reflectance spectroscopy. ${ }^{5,10}$ Because the optical anisotropy directly reflects the energy-band structures of emitting layers, critical factors to be investigated in strained-QW-based light emitters are strain and quantum confinement. Their effects have been theoretically studied for arbitrary crystalline orientations of $\mathrm{GaN} \mathrm{QWs}^{12}$ and $\mathrm{GaN} / \mathrm{Al}_{x} \mathrm{Ga}_{1-x} \mathrm{~N}$ QWs. ${ }^{13}$ Experimentally, strain effects have been examined for nonpolar $\{1 \overline{1} 00\}$ (Refs. 14 and 15) and polar (0001) (Refs. 16 and 17) GaN films. Regarding $\operatorname{In}_{x} \mathrm{Ga}_{1-x} \mathrm{~N}$ QWs with relatively low In compositions, the polarization properties have been investigated for semipolar $\{11 \overline{2} 2\}$ (Ref. 5) and $\{1 \overline{1} 01\},{ }^{18}$ and nonpolar (1 $\left.\overline{1} 00\right)$ (Refs. 19-21) by PL and electroluminescence (EL) spectroscopy. The results consistently showed that the emission is polarized perpendicular to the $c$ axis. In contrast, herein, we revealed polarization switching phenomena in semipolar $\{11 \overline{2} 2\} \operatorname{In}_{x} \mathrm{Ga}_{1-x} \mathrm{~N} / \mathrm{GaN}$ QWs and LEDs through systematic studies on these structures with various well widths and In compositions. Then, the experimental results were analyzed by the $\boldsymbol{k} \cdot \boldsymbol{p}$ approach to gain some insight into the energyband structures of semipolar or nonpolar $\operatorname{In}_{x} \mathrm{Ga}_{1-x} \mathrm{~N}$ QW.

The QWs and LEDs were fabricated by metalorganic vapor phase epitaxy on $\{11 \overline{2} 2\}$ GaN bulk substrates. The growth and sample structures are detailed in Refs. 5 and 6.
PACS number(s): 78.67.De, 78.55.Cr, 78.60.Fi

The $\mathrm{In}_{x} \mathrm{Ga}_{1-x} \mathrm{~N}$ well widths were designed by referring to the growth rate, which was derived in advance from X-ray diffraction profiles of multiple QWs. The In compositions were determined by the well width and the PL or EL peak energy. PL and EL were measured at room temperature (RT). PL was excited by a He-Cd laser ( $325 \mathrm{~nm}$ ), while EL was driven by a direct-current source. The excitation was as weak as $12.7 \mathrm{~W} / \mathrm{cm}^{2}$ for PL and $0.5 \mathrm{~A} / \mathrm{cm}^{2}$ for EL, unless stated. The in-plane polarization properties were assessed using a Glan-Taylor polarizer. For EL, microscopy was adopted to limit the observation area to $\phi \sim 10 \mu \mathrm{m}$ (in-plane) and $\sim 14 \mu \mathrm{m}$ (out-of-plane) using a receiving pin hole of $\phi 62.5 \mu \mathrm{m}$. This procedure enabled the influence of light scattering within the LED chips and dies to be eliminated.

In terms of the in-plane optical anisotropy in the $\{11 \overline{2} 2\}$ plane, the key directions are the $[1 \overline{1} 00]$ direction $(\perp[0001])$ and the perpendicular [1-123] direction. Figures 1(a) and 1(b) show typical polarization EL spectra of $(11 \overline{2} 2)$ blue and red LEDs, respectively. The In composition and well width for the blue LED were $17 \%$ and $2.4 \mathrm{~nm}$, respectively, while those for the red LED were $48 \%$ and $2.9 \mathrm{~nm}$. The spectra were acquired for the electric-field vectors of emitted light $(\boldsymbol{E})$ parallel to the two key directions, where the EL intensity reached either a minimum or a maximum. For the blue LED, the EL intensity was maximized with $\boldsymbol{E} \|[1 \overline{1} 00]$ and minimized with $\boldsymbol{E} \|[\overline{1} \overline{1} 23]$. This predominant polarization perpendicular to the $c$ axis is similar to the previous reports. ${ }^{5,18-21}$ On the contrary, the red LED demonstrated the opposite tendencies, suggesting considerable modifications of the energy-band structures. To survey the polarization properties in $\{11 \overline{2} 2\}$ semipolar QWs and LEDs, polarization degrees, $\rho$, were estimated for all fabricated samples. $\rho$ was defined as $\left(I_{[1 \overline{1} 00]}-I_{[\overline{1} 123]}\right) /\left(I_{[1 \overline{1} 00]}+I_{[\overline{11} 23]}\right)$, using the integrated luminescence intensity, $I$. Therefore, the positive (negative) $\rho$ means the predominant polarization along the [1 $\overline{1} 00]$ ([11123]) direction. Figure 1(c) displays the sign of $\rho$ as a function of In composition and $\operatorname{In}_{x} \mathrm{Ga}_{1-x} \mathrm{~N}$ thickness. The solid curve in Fig. 1(c) shows the calculated critical layer thickness for $c$-plane $\operatorname{In}_{x} \mathrm{Ga}_{1-x} \mathrm{~N}$ on unstrained $\mathrm{GaN}{ }^{22}(\mathrm{Be}-$ cause relaxation processes in semipolar films have yet to be clarified, the model for the $c$ plane was tentatively adapted. The QWs with $\sim 50 \%$ In are thicker than this critical thick- 

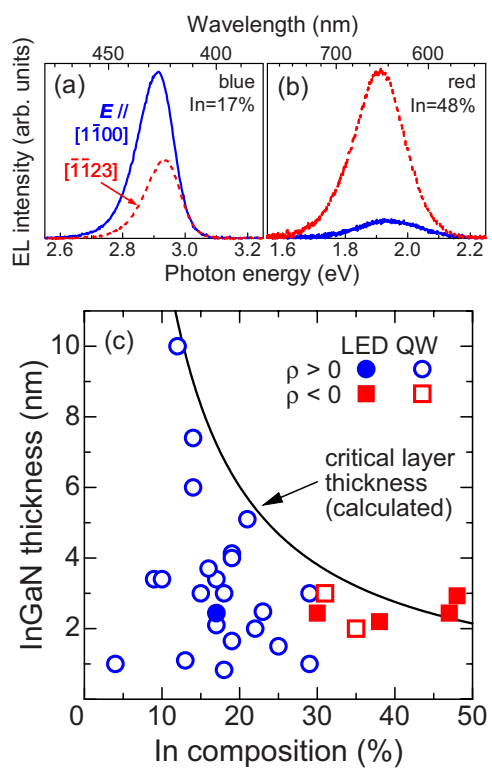

FIG. 1. (Color online) Polarization EL spectra of (11 $\overline{2} 2$ ) (a) blue and (b) red LEDs driven at $0.5 \mathrm{~mA}$. The (blue/dark gray) solid lines show EL spectra with $\boldsymbol{E} \|[1 \overline{1} 00]$, while the (red/gray) broken lines are for $\boldsymbol{E} \|[\overline{1} 123]$. (c) Sign of the polarization degree $(\rho)$ as a function of $\operatorname{In}_{x} \mathrm{Ga}_{1-x} \mathrm{~N}$ thickness and In composition.

ness, but the polarization properties were consistent with other samples, suggesting the absence of relaxation.) As long as $\mathrm{In}_{x} \mathrm{Ga}_{1-x} \mathrm{~N}$ was pseudomorphically grown on $\mathrm{GaN}$, an In composition less than $\sim 30 \%$ caused polarization parallel to $[1 \overline{1} 00](\rho>0)$, while a composition greater than $\sim 30 \%$

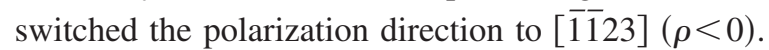

Comparison between Figs. 1(a) and 1(b) revealed that the EL spectra with $\boldsymbol{E} \|[1 \overline{1} 00]$ and $\boldsymbol{E} \|[\overline{1} \overline{1} 23]$ are located at different energies. Figure 2 shows the energy separations as a function of In composition, where the peak energy for $\boldsymbol{E} \|[1 \overline{1} 00]\left(E_{\mathrm{P}}^{[1 \overline{1} 00]}\right)$ was subtracted from that for $\boldsymbol{E} \|[\overline{1} \overline{1} 23]$ $\left(E_{\mathrm{P}}^{[\overline{1123]})}\right.$. As discussed below, the solid curve is due to the fit. Clearly, for an In composition less than $\sim 30 \%$, spectra with $\boldsymbol{E} \|[1 \overline{1} 00]$ were located at lower energies, but the opposite tendencies were observed with a composition greater than $\sim 30 \%$. Comparing Fig. 2 to Fig. 1(c) indicated that the direction for lower energy emissions corresponds to the predominantly polarizing direction; namely, for the [1 $\overline{1} 00]$ polarization $(\rho>0), E_{\mathrm{P}}^{[1100]}<E_{\mathrm{P}}^{[\overline{11} 23]}$, whereas for the $[\overline{1} 123]$ polarization $(\rho<0), E_{\mathrm{P}}^{[\overline{11} 23]}<E_{\mathrm{P}}^{[1 \overline{1} 00]}$. These observations suggested that the polarization properties are determined by two competing components related to $[1 \overline{1} 00]$ and $[\overline{1} \overline{1} 23]$.

To identify the two components, the injection current dependence of EL spectra was measured for a (1122) green LED with an In composition of $38 \%$ and a well width of 2.2 nm, as shown in Fig. 3. Two findings should be emphasized: (i) Weaker EL spectra were always located at higher energies, which is consistent with Figs. 1(a) and 1(b); (ii) As the injection current increased, the EL intensity for $\boldsymbol{E} \|[1 \overline{1} 00]$ relative to that for $\boldsymbol{E} \|[\overline{1} 123]$ increased, but the peak energy separation remained nearly constant.

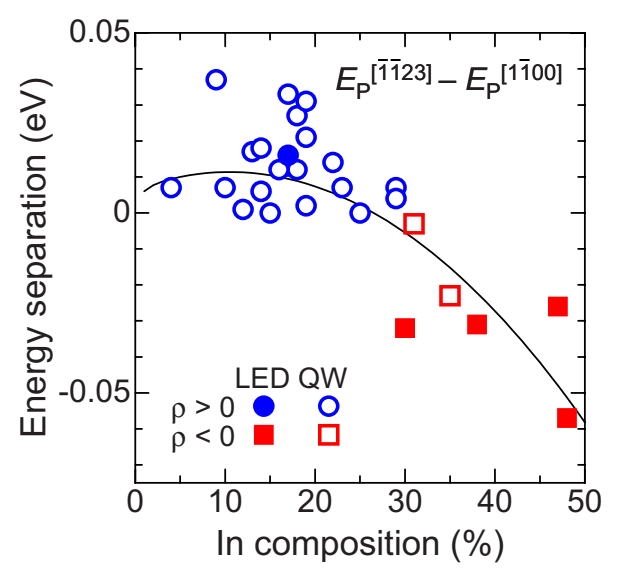

FIG. 2. (Color online) Energy separation between the emission peaks of spectra for $\boldsymbol{E} \|[1 \overline{1} 00]$ and $\boldsymbol{E} \|[\overline{1} \overline{1} 23]$ as a function of In composition. Solid curve is a result of the fit.

All the above results can well be interrelated by considering the $\mathrm{A}$ and $\mathrm{B}$ valence bands. ${ }^{23}$ When the In composition was lower than $\sim 30 \%$, the topmost A band was dominated by a $P$ orbit along the $[1 \overline{100}]$ direction, while the second highest $\mathrm{B}$ band was dominated by that along the [1-123] direction. As the In composition increased, these two valence bands approached, and eventually, a crossover occurred, which resulted in the $\mathrm{A}$ and $\mathrm{B}$ bands being dominated by the [11-23]-related and [1 100$]$-related states, respectively. Figure 4 is a schematic of this variation in the band alignments. The observed energy separations (Fig. 2) well approximated those between the A and B bands. On the other hand, because the hole population in the A band was inevitably greater than that in the B band, A-band-related emissions were always more intense. As carrier injection increased, the quasi-Fermi level for holes approached the valence band. Consequently, the population in the $\mathrm{B}$ band increased with respect to that in the A band, which relatively strengthened the B-band-related emission without remarkably changing the peak energy separation (Fig. 3).

Because carriers were thermally redistributed in the A and $\mathrm{B}$ bands under the current experiments at RT, differences in

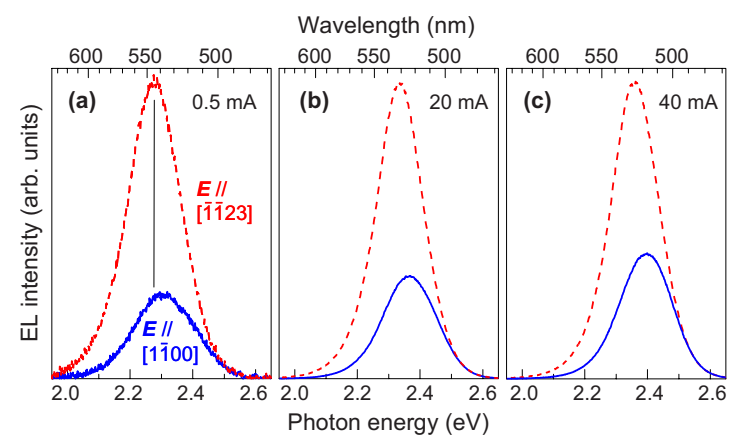

FIG. 3. (Color online) Injection current dependence of polarization EL spectra of a (11/22) green LED. The (blue/dark gray) solid and (red/gray) broken lines were defined in the same manner as those in Fig. 1. Thin solid line shows the energy difference between EL spectra with $\boldsymbol{E} \|[1 \overline{1} 00]$ and $\boldsymbol{E} \|[\overline{1} \overline{1} 23]$. 


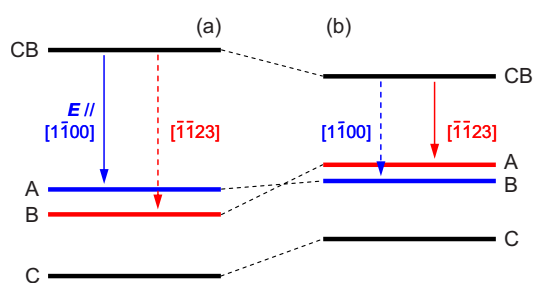

FIG. 4. (Color online) Band alignments of $\{11 \overline{2} 2\}$ QWs with (a) In $\leqslant 30 \%$ and (b) In $\geq 30 \%$. CB stands for the conduction band.

the properties of A and B excitons such as thermal stability may influence the relative intensities of polarization spectra acquired for the $[1 \overline{1} 00]$ and [ $\overline{1} \overline{1} 23]$ directions. Therefore, to separately assess the polarization properties of A and B bands, each polarization spectrum was assumed to involve two emission components due to those bands, and was decomposed into two Gaussian curves. Hence, there were totally four Gaussian curves for a pair of polarization spectra. However, because the A-band-related (or B-band-related) component in either polarization spectrum must have the identical peak position and line width, the number of fitting parameters was reduced to eight. Furthermore, from the stronger polarization spectrum, the peak position, line width, and amplitude of the A-band-related emission could be determined without serious errors. This, in turn, contributed to a better fit of the B-band-related parameters, although the much weaker B-band-related emission sometimes caused an uncertainty of at most $20 \%$ particularly for QWs with large A-B splitting energies. Using thus determined A and B band components, the polarization degree was evaluated for each band.

Figure 5 displays the well-width dependence of the polarization degrees for the A and B bands. The QWs were grown under the same conditions, and the In composition was $20 \pm 2 \%$. The polarization degrees were nearly independent of the well thickness, and $\sim 0.8$ for the A band and -1.0 for the B band. These quantities indicated that both emissions are polarized almost linearly along the perpendicular two directions, strikingly different from bulk GaN ( $\rho=0.56$ for A and 0 for $\mathrm{B}$ ) due to the mismatch-induced strain. A theoretical study has predicted the degradation of linear polarization in extremely thin QWs, ${ }^{12}$ but the prediction was not observed experimentally within the current experimental accuracy. This discrepancy should be due to the infinite-barrier approximation adopted in the calculation.

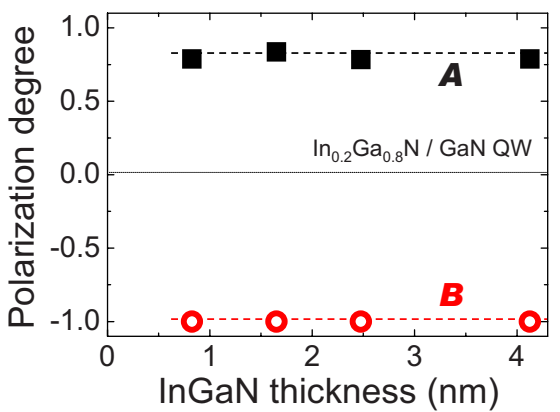

FIG. 5. (Color online) $\operatorname{In}_{x} \mathrm{Ga}_{1-x} \mathrm{~N}$ thickness dependence of the polarization degrees for the $\mathrm{A}$ and $\mathrm{B}$ bands.

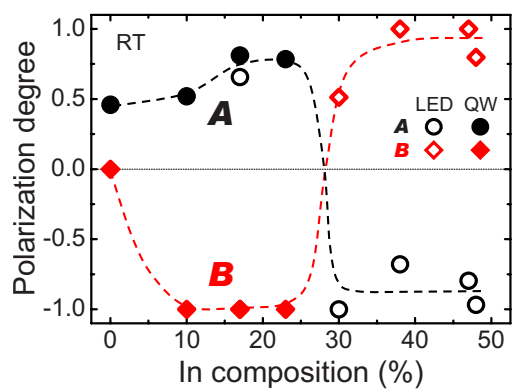

FIG. 6. (Color online) In composition dependence of the A-band and B-band polarizations.

For the same series of the QWs, the A-band polarization degrees were directly evaluated by lowering the measurement temperature to $10 \mathrm{~K}$, where carriers were hardly populated to the B band. Those were $0.83,0.84$, and 0.81 for well widths of $0.8,1.7$, and $5.1 \mathrm{~nm}$, respectively, consistent with Fig. 5. Furthermore, although the large inhomogeneous broadening of PL and EL suggests the presence of localized excitons, the polarization degrees were nearly constant within the spectra, irrespective of the well widths, which indicates that the carrier localization does not affect the polarization properties.

On the other hand, Fig. 6 shows the In-composition dependence of the polarization degrees for the $\mathrm{A}$ and $\mathrm{B}$ bands in QWs and LEDs with 2-3-nm-thick $\operatorname{In}_{x} \mathrm{Ga}_{1-x} \mathrm{~N}$. When the In composition was increased from 0 to $\sim 25 \%$, the polarization degree for the A band was gradually increased from $\sim 0.5$ to 0.8 and that for the B band was drastically changed from nearly 0 for $\mathrm{In}=0 \%(\mathrm{GaN})$ to about -1.0 for $\mathrm{In}$ $=10-25 \%$. Further increasing the In composition caused a polarization switch at $\sim 30 \%$. For the In composition above $\sim 30 \%$, the polarization degree for the A (B) band was $-0.8(0.9)$.

Let us discuss the mechanism of the polarization switch, based on the $\boldsymbol{k} \cdot \boldsymbol{p}$ approach. The $A$ and $D$ parameters are generally important to describe QW properties, as A's express the effective masses, and $D$ 's are the deformation potentials. However, as long as we examined the reported, widely varied $A$ parameters, ${ }^{24}$ such scattering has a negligible effect on the calculated polarization properties, indicating that, in highly strained $\operatorname{In}_{x} \mathrm{Ga}_{1-x} \mathrm{~N} / \mathrm{GaN}$ QWs, the quantum confinement does not affect the valence-band order so significantly as the strain does through the deformation potentials. This finding is consistent with Figs. 5 and 6. Hence, the quantum confinement effect was neglected in the following analysis. Further neglecting the spin-splitting interaction, the $6 \times 6$ valence-band Hamiltonian can be diagonalized to obtain the eigenenergy for each valence band. The energy separation between the $\mathrm{A}$ and $\mathrm{B}$ bands at the $\Gamma$ point in strained $\operatorname{In}_{x} \mathrm{Ga}_{1-x} \mathrm{~N}$ layers with arbitrary orientations can thus be simplified to

$$
E^{X^{\prime}}-E^{Y^{\prime}}=\left\{E_{1}-3 E_{2}-\sqrt{\left(E_{1}+E_{2}\right)^{2}+8\left(D_{6} \epsilon_{x z}\right)^{2}}\right\} / 2,
$$

where $E^{X^{\prime}}\left(E^{Y^{\prime}}\right)$ is the transition energy for the $X^{\prime}\left(Y^{\prime}\right)$ polarization, $E_{1}=\Delta_{\mathrm{cr}}+D_{3} \epsilon_{z z}+D_{4}\left(\epsilon_{x x}+\epsilon_{y y}\right), E_{2}=D_{5}\left(\epsilon_{x x}-\epsilon_{y y}\right), \Delta_{\mathrm{cr}}$ is the crystal-field splitting energy, and $\epsilon$ represents strain 
tensor elements. ${ }^{25} X^{\prime}$ and $Y^{\prime}$ stand for the two in-plane perpendicular directions, and $Y^{\prime} \perp$ [0001]. Therefore, $E^{X^{\prime}}$ and $E^{Y^{\prime}}$ are related to the A or B band. For the (11) 2 ) plane, $X^{\prime}$ was [1-123], and $Y^{\prime}$ was [1 100$]$. The $(x, y, z)$ orthogonal coordinates were defined with respect to the wurtzite lattice, where $x$ and $y$ were in the $c$ plane, while $z$ was along the $c$ axis. Moreover, $y$ was the rotation axis, that is, $y \|[1 \overline{1} 00]$ for $(11 \overline{2} 2)$ planes. Semipolar planes are characterized by anisotropic strain in the $c$ plane $\left(\epsilon_{x x} \neq \epsilon_{y y}\right)$ and the presence of shear strain $\left(\epsilon_{x z} \neq 0\right)$, which allow the contributions from $D_{5}$ and $D_{6}$ and promote band mixing.

Initially we calculated Eq. (1) using the well-accepted physical parameters. ${ }^{26}$ The $\operatorname{In}_{x} \mathrm{Ga}_{1-x} \mathrm{~N}$ parameters were derived by the linear interpolation between the $\mathrm{GaN}$ and $\mathrm{InN}$ parameters, and the strain was calculated assuming the coherent growth. However, this parameter set could not reproduce the polarization switch probably due to the lack of data for the InN deformation potentials, which were substituted by those of $\mathrm{GaN}$. Therefore, the $\mathrm{InN}$ deformation potentials $\left(D_{3}, D_{4}, D_{5}\right.$, and $\left.D_{6}\right)$ were determined so as to minimize the difference between the experimental data in Fig. 2 and calculation by Eq. (1) under the cubic approximation of $D_{4}=$ $-D_{3} / 2 .^{27}$ (This equation was experimentally confirmed for wurtzite $\mathrm{GaN}^{14}$ ) In the fit, the experimental data for
$\operatorname{In}_{x} \mathrm{Ga}_{1-x} \mathrm{~N}$ thicker than $2 \mathrm{~nm}$ was used to surely avoid the influence of the quantum confinement. Consequently, $D_{4}=$ $-D_{3} / 2=-0.05 \mathrm{eV}, D_{5}=-3.5 \mathrm{eV}$, and $D_{6}=-8.8 \mathrm{eV}$ were derived. Although $D_{1}$ and $D_{2}$ cannot be determined as they are not involved in Eq. (1), we suggest $D_{2}-D_{1}=D_{3}$ $=0.10 \mathrm{eV}$, applying another cubic approximation. The result of this fit is shown as a solid curve in Fig. 2, which agrees reasonably well with the experimental data.

The energy differences of $E^{X^{\prime}}-E^{Y^{\prime}}$ in nonpolar $\mathrm{In}_{x} \mathrm{Ga}_{1-x} \mathrm{~N} / \mathrm{GaN}$ QWs were reported to be 5, 40, and $60 \mathrm{meV}$ for In compositions of 10,18 , and $23 \%$, respectively, ${ }^{21}$ which we evaluated from the reported well width and emission wavelength. On the other hand, the energy differences evaluated by Eq. (1) with the parameters in Ref. 26 were 19, 25, and $30 \mathrm{meV}$ for the respective In compositions, while the obtained InN deformation potentials provided a much better fit of 24, 43, and $59 \mathrm{meV}$, supporting the current analysis. It is interesting to note that neither parameter set predicts polarization switch in nonpolar QWs. Therefore, the determinant of the polarization switching properties in semipolar QWs is the shear strain, $\epsilon_{x z}$, which is absent in nonpolar QWs. Regarding a device application, the present results indicate that cavity mirrors of semipolar laser diodes emitting green or a longer wavelength can be formed by cleavage. *funato@kuee.kyoto-u.ac.jp

${ }^{1}$ V. Fiorentini, F. Bernardini, F. Della Sala, A. Di Carlo, and P. Lugli, Phys. Rev. B 60, 8849 (1999).

${ }^{2}$ P. Waltereit, O. Brandt, A. Trampert, H. T. Grahn, J. Menniger, M. Ramsteiner, M. Reiche, and K. H. Ploog, Nature (London) 406, 865 (2000).

${ }^{3}$ B. A. Haskell, F. Wu, S. Matsuda, M. D. Craven, P. T. Fini, S. P. DenBaars, J. S. Speck, and S. Nakamura, Appl. Phys. Lett. 83, 1554 (2003).

${ }^{4}$ K. Nishizuka, M. Funato, Y. Kawakami, Sg. Fujita, Y. Narukawa, and T. Mukai, Appl. Phys. Lett. 85, 3122 (2004).

${ }^{5}$ M. Ueda, K. Kojima, M. Funato, Y. Kawakami, Y. Narukawa, and T. Mukai, Appl. Phys. Lett. 89, 211907 (2006).

${ }^{6}$ M. Funato, M. Ueda, Y. Kawakami, Y. Narukawa, T. Kosugi, M. Takahashi, and T. Mukai, Jpn. J. Appl. Phys., Part 2 45, L659 (2006).

${ }^{7}$ A. Tyagi, H. Zhong, N. N. Fellows, M. Iza, J. S. Speck, S. P. DenBaars, and S. Nakamura, Jpn. J. Appl. Phys., Part 2 46, L129 (2007)

${ }^{8}$ S.-H. Park and S.-L. Chuang, Phys. Rev. B 59, 4725 (1999).

${ }^{9}$ T. Takeuchi, H. Amano, and I. Akasaki, Jpn. J. Appl. Phys., Part 1 39, 413 (2000).

${ }^{10}$ K. Kojima, M. Ueda, M. Funato, and Y. Kawakami, Phys. Status Solidi B 244, 1853 (2007).

${ }^{11}$ B. Gil, Appl. Phys. Lett. 90, 121903 (2007).

${ }^{12}$ A. A. Yamaguchi, Jpn. J. Appl. Phys., Part 2 46, L789 (2007).

${ }^{13}$ C.-N. Chen, S.-H. Chang, M.-L. Hung, J.-C. Chiang, I. Lo, W.-T. Wang, M.-H. Gau, H.-F. Kao, and M.-E. Lee, J. Appl. Phys. 101, 043104 (2007).
${ }^{14}$ B. Gil and A. Alemu, Phys. Rev. B 56, 12446 (1997).

${ }^{15}$ S. Ghosh, P. Waltereit, O. Brandt, H. T. Grahn, and K. H. Ploog, Phys. Rev. B 65, 075202 (2002).

${ }^{16}$ B. Gil, F. Hamdani, and H. Morkoç, Phys. Rev. B 54, 7678 (1996).

${ }^{17}$ A. Alemu, B. Gil, M. Julier, and S. Nakamura, Phys. Rev. B 57, 3761 (1998).

${ }^{18}$ M. Feneberg, F. Lipski, R. Sauer, K. Thonke, P. Brückner, B. Neubert, T. Wunderer, and F. Scholz, J. Appl. Phys. 101, 053530 (2007).

${ }^{19}$ Y. J. Sun, O. Brandt, M. Ramsteiner, H. T. Grahn, and K. H. Ploog, Appl. Phys. Lett. 82, 3850 (2003).

${ }^{20}$ S. Nakagawa, H. Tsujimura, K. Okamoto, M. Kubota, and H. Ohta, Appl. Phys. Lett. 91, 171110 (2007).

${ }^{21}$ H. Masui, H. Yamada, K. Iso, S. Nakamura, and S. P. DenBaars, Appl. Phys. Lett. 92, 091105 (2008).

${ }^{22}$ A. Fischer, H. Kühne, and H. Richter, Phys. Rev. Lett. 73, 2712 (1994).

${ }^{23}$ In strained $\operatorname{In}_{x} \mathrm{Ga}_{1-x} \mathrm{~N}$, the $|X \pm i Y\rangle$-like and $|Z\rangle$-like hole states are mixed, and therefore, the valence bands are simply denoted as $A, B$, and $C$ from the top in this study.

${ }^{24}$ D. Fritsch, H. Schmidt, and M. Grundmann, Phys. Rev. B 67, 235205 (2003), and references therein.

${ }^{25}$ K. Kojima, H. Kamon, M. Funato, and Y. Kawakami, Phys. Status Solidi C 5, 3038 (2008).

${ }^{26}$ I. Vurgaftman and J. R. Meyer, J. Appl. Phys. 94, 3675 (2003).

${ }^{27}$ G. L. Bir and G. E. Pikus, Symmetry and Strain-Induced Effects in Semiconductors (Wiley, New York, 1974). 